\title{
Constraining the contribution of Gamma-Ray Bursts to the high-energy diffuse neutrino flux with 10 years of ANTARES data
}

Angela Zegarelli ${ }^{a, b, *}$ and Silvia Celli ${ }^{a, b}$ on behalf of the ANTARES Collaboration (a complete list of authors can be found at the end of the proceedings)

a Dipartimento di Fisica, Università "Sapienza",

P.le Aldo Moro 2, I-00185 Roma, Italy

${ }^{b}$ INFN, Sezione di Roma,

P.le Aldo Moro 2, I-00185 Roma, Italy

E-mail: angela.zegarelli@roma1.infn.it, silvia.celli@roma1.infn.it

Addressing the origin of the observed diffuse astrophysical neutrino flux is one of the main challenges in the context of the neutrino astronomy nowadays. Among several astrophysical sources, Gamma-Ray Bursts (GRBs) are considered interesting candidates to be explored. Indeed, being the most powerful explosions observable in the Universe, they are potentially able to achieve the energetics required to reproduce the neutrino flux. Thus, they are expected to provide at least some contribution to the astrophysical diffuse neutrino flux. Within the framework of the fireball model, mesons can be produced during photo-hadronic interactions occurring in the internal shocks between shells emitted by the central engine; from their decays, high-energy gamma rays and neutrinos are expected to be generated. Within this scenario, the results of a stacked search for astrophysical muon neutrinos performed in space and time coincidence with 784 GRBs in the period 2007-2017 using ANTARES data are presented. The neutrino flux expectation from each GRB detectable by ANTARES was calculated in the framework of the classical internal shock model. Given the absence of coincident neutrinos, the contribution of the detected GRB population to the neutrino diffuse flux is constrained to be less than $10 \%$ around $100 \mathrm{TeV}$. In addition, the systematic uncertainties on the diffuse flux are computed by propagating to the stacked limit the uncertainties on the model parameters for each individual burst.

$37^{\text {th }}$ International Cosmic Ray Conference (ICRC 2021)

July 12 th - 23rd, 2021

Online - Berlin, Germany

\footnotetext{
${ }^{*}$ Presenter
} 


\section{Introduction}

High-energy astrophysical neutrinos have been discovered few years ago [1], opening a new window to the study of the Universe. Identifying the sources of these neutrinos is one of the key scientific targets of the astroparticle physics community nowadays.

Among several astrophysical sources, Gamma-Ray Bursts (GRBs), the most powerful known explosions in the Universe (with energy release between $10^{51}$ and $10^{54} \mathrm{ergs}$ in few seconds) [2], are considered one of the most promising candidate sources of astrophysical neutrinos. Indeed, within the framework of a hadronic scenario, if hadrons are accelerated in GRBs, neutrinos are expected to be produced by the interactions between protons (or heavier nuclei) and the intense radiation field of the jet. Multi-messenger searches targeted at GRBs appear very promising; being transients and extremely energetic explosions, GRBs allow to strongly reduce the background accumulated during their very short duration. In addition, neutrinos, being electrically neutral, stable and weakly interacting particles, are ideal messengers in the search for distant astrophysical objects. Thus, unlike protons or charged nuclei, they are not diverted in their path from their source to the Earth. Furthermore, unlike photons, neutrinos are not absorbed while propagating towards the Earth. For these reasons, searching for a temporal and spatial coincidence of GRB photons and high-energy neutrinos is crucial to safely identify this kind of sources as hadronic factories and to shed light on the composition of their jets.

Over the past years, the neutrino telescopes of the Northern and Southern hemispheres, respectively ANTARES [3] and IceCube [4], have been searching for neutrino signals coincident with GRBs in time and direction (see [5, 6] for previous ANTARES studies on GRBs and [7-9] for IceCube). The lack of detections from these searches has allowed to set progressively stronger upper limits, thus limiting also the possible contribution of these sources to the observed astrophysical diffuse neutrino flux. Nonetheless, current limits do not yet provide significant constraints on the validity of the internal shock model, once the many uncertainties on the several parameters that affect the predictions are taken into account.

For this reason, the innovative search for astrophysical muon neutrinos from GRBs, already presented in [10], is here summarised. This work focuses on improving the predictions on the expected neutrino fluences from GRBs, by considering the wealth of information accumulated so far thanks to the many astronomical observations, rather than assuming some fixed standard values that do not correctly reproduce the properties of the source sample. Contextually, the different uncertainties due to the poor knowledge of the source dynamics are taken into account and propagated on the produced neutrino spectrum, with the aim of providing a clear understanding of the assumptions and limitations behind the set upper limits.

\section{GRB selection and parameters}

In order to reduce the very abundant background coming from atmospheric muons, up-going track-like events are used in this analysis, hence only GRBs arisen below the ANTARES horizon at trigger time have been selected. 


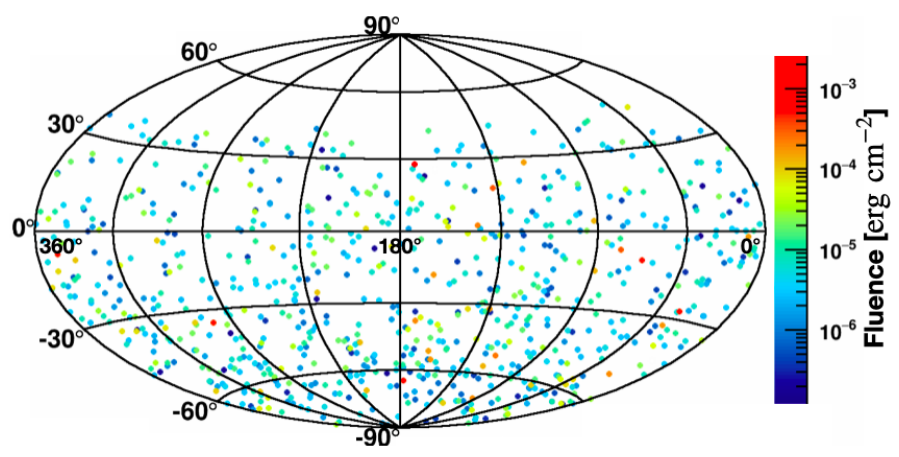

Figure 1: Sky distribution and fluence of the selected 784 GRBs in equatorial coordinates.

A sample of 784 long GRBs $\left(\mathrm{T}_{90} \geq 2 \mathrm{~s}\right)^{1}$, occurred in the years 2007-2017, is considered in the present analysis (their spatial distribution in the equatorial sky is shown in Fig. 1).

The GRB parameters needed for the search (time, direction) and the simulation of expected neutrino fluxes, e.g. photon spectrum, fluence and redshift, are collected from published results of Swift $^{2}$, Fermi $^{3}$ and Konus-Wind ${ }^{4}$. For more details regarding the selection criteria see [10].

\section{Computation of the neutrino flux from internal shocks and its uncertainties}

The neutrino fluxes expected for each GRB of the sample have been computed by the event generator 'Neutrinos from Cosmic Accelerator' (NeuCosmA) [12, 13], which operates within the framework of the fireball model [14], namely the most commonly accepted scenario to explain the physics of GRBs. In such a model, mesons can be produced during photo-hadronic interactions occurring in the internal shocks between shells emitted by the central engine; from their decays, high-energy gamma rays and neutrinos are expected to be generated. These processes constitute the so-called prompt phase of the emission. Nonetheless, if GRBs were purely leptonic sources, the observed radiation would be completely ascribed to processes involving primary electrons, such that there would be no possibility to produce neutrinos in these sources.

The neutrino fluxes computed with NeuCosmA are normalised through several quantities, including the intensity of the photon flux and the ratio between the fireball energy going into protons with respect to that going into electrons, the so-called baryonic loading, $f_{\mathrm{p}}$. Note that the latter parameter is an unknown of the problem, possibly constrained by neutrino observations. From the theoretical point of view, a reasonable value for it could be $f_{\mathrm{p}} \simeq 10$ [13], which will be considered fixed for each GRB of this analysis. The adopted version of NeuCosmA assumes a one-zone collision, namely it simulates average shell properties, such as an average shock speed or Lorentz factor $\Gamma$ (i.e. the bulk Lorentz factor of the jet). A single representative collision is realised

\footnotetext{
${ }^{1} \mathrm{~T}_{90}$ is the time in which $90 \%$ of the gamma-ray fluence is emitted, during the so-called prompt phase.

${ }^{2}$ Swift catalogue in https://swift.gsfc.nasa.gov/archive/grb_table/

${ }^{3}$ Fermi-GBM in https://heasarc . gsfc . nasa.gov/W3Browse/fermi/fermigbrst. html. Fermi-LAT in [11].

${ }^{4}$ Konus-Wind information is only available through the GCN archive: http://gcn.gsfc.nasa.gov/gcn3_ archive.html
} 


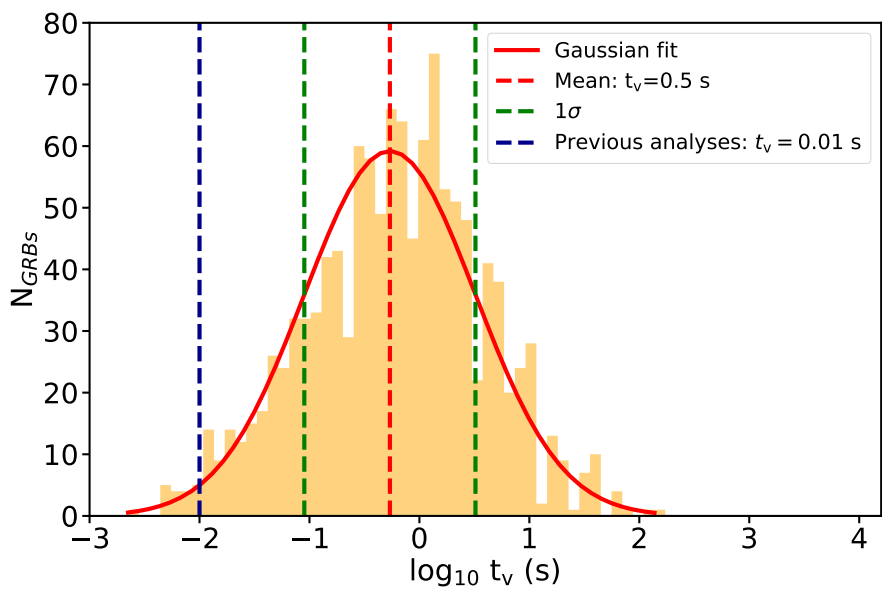

Figure 2: Distribution of minimum variability timescales obtained analysing 1213 GRB light curves [1618]. The solid red line indicates the Gaussian fit of the distribution. The dashed red line is the mean of the distribution, from which a mean value of $t_{\mathrm{v}}=0.5 \mathrm{~s}$ is obtained. The dashed green lines indicate the standard deviation of the distribution. The dashed blue indicates the default value $t_{\mathrm{v}}=10 \mathrm{~ms}$, previously adopted e.g. in [5] and [9].

at the so-called internal shock radius, located at a distance

$$
\mathrm{R}_{\mathrm{is}} \simeq \frac{2 \Gamma^{2} c t_{\mathrm{v}}}{(1+z)} \simeq 2 \times 10^{13}\left(\frac{t_{v}}{0.01 \mathrm{~s}}\right)\left(\frac{\Gamma}{10^{2.5}}\right)^{2}\left(\frac{3}{1+z}\right) \mathrm{cm}
$$

from the central emitter. Note that the internal shock radius strongly affects the characteristic energy range of emitted neutrinos, while simultaneously scaling the normalisation of the neutrino spectrum. In addition, as Eq. (1) shows, it depends on some intrinsic parameters of the emission regions, like the boost Lorentz factor $\Gamma$, and the minimum variability timescale $t_{v}$, and it also requires the knowledge of redshift of sources. All these parameters are difficult to determine, since the former cannot reliably be figured out on a source-by-source basis and, regarding the latter, the host galaxy of the GRB can often fail to be identified by the multi-wavelength follow-up. To overcome such uncertainties, instead of using default benchmark values, a novel technique that allows to infer them is here presented. In particular, for the Lorentz factor, its correlation with the mean isotropic gamma-ray luminosity $\mathrm{L}_{\gamma, \text { iso }}$, as found by [15] is used:

$$
\Gamma \simeq 249\left(\frac{\mathrm{L}_{\gamma, \text { iso }}}{10^{52} \mathrm{erg} / \mathrm{s}}\right)^{0.30} .
$$

However, the application of this method is not free from uncertainties, as the isotropic luminosity is also often unknown, given that it requires the knowledge of the redshift (because of the luminosity distance $d_{L}=d_{L}(z)$ ). As redshift is only known in $11 \%$ of the cases, a method accounting for the observed redshift distribution of long GRBs was applied in order to estimate respectively i) the luminosity distance, ii) the isotropic gamma-ray luminosity and iii) the bulk Lorentz factor, for each GRB in the selected sample. Specifically, 1000 random extractions of the $z$ value are performed for GRBs with unknown $z$, according to the redshift distribution of long GRBs, as observed by 
Swift since 2005 until now ${ }^{5}$. A similar procedure of random extraction according to a known distribution of values is adopted for the minimum variability timescale $t_{\mathrm{v}}$, that is known only in the $33 \%$ of the cases. For this reason, a distribution of estimated values of $t_{\mathrm{v}}$ for long GRBs is built, as shown in Fig. 2 [16-18]. Hence, by using the extracted values of $z$ and $t_{\mathrm{v}}, 1000$ fluxes for each GRB (for which $z$ and/or $t_{\mathrm{v}}$ are unknown) are simulated, in order to estimate the average neutrino fluence expected from each GRB, and its uncertainty obtained by spanning the unknown parameter values over their allowed ranges. The method allows also to investigate how these uncertainties affect the neutrino spectra and to identify the parameter that contributes the most. As the neutrino flux is expected to be extremely sensitive to the Lorentz factor [19], a treatment of the additional systematics associated with adopting a different method for deriving $\Gamma$ (e.g. [20]) is also presented in [10].

As result of such a procedure, it has been observed that (i) $\Gamma$ is the parameter which impacts the most the GRB-neutrino flux predictions, (ii) $t_{\mathrm{v}}$ contributes more than redshift to the uncertainty on the neutrino flux predictions from GRBs. Indeed, when letting $t_{\mathrm{v}}$ free to vary, the estimated uncertainty on the neutrino flux expected from the model spans up to several orders of magnitude.

\section{Stacked muon neutrino fluence from 784 GRBs}

By summing over all the individual neutrino fluences, the total fluence $\mathrm{E}_{v_{\mu}}^{2} \mathrm{~F}_{v_{\mu}} \pm 2 \sigma$ expected from the cumulative contribution of the selected 784 GRBs in the period 2007-2017 is calculated (see Fig. 3). The quasi-diffuse neutrino flux $\mathrm{E}_{v_{\mu}}^{2} \phi_{v_{\mu}} \pm 2 \sigma$ induced by the same sources is shown too; it is obtained by rescaling the total fluence with the average expected rate of long GRBs distributed over the full sky, following previous analogous studies [5, 7-9].

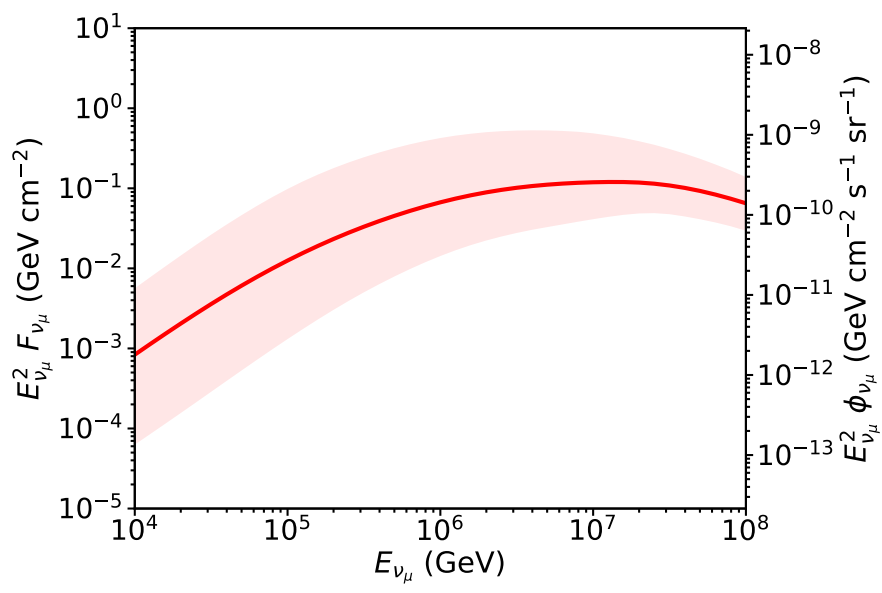

Figure 3: Total neutrino fluence $\mathrm{E}_{\nu_{\mu}}^{2} \mathrm{~F}_{\nu_{\mu}}$ expected from the $784 \mathrm{GRBs}$ in the sample selected in the period 2007-2017 (left-hand axis) and corresponding quasi-diffuse neutrino flux $\mathrm{E}_{v_{\mu}}^{2} \phi_{\nu_{\mu}}$ (right-hand axis). The shaded region indicates the error band, obtained from the sum of the individual maximum $\left(\mathrm{E}_{\nu_{\mu}}^{2} \mathrm{~F}_{v_{\mu}}+2 \sigma\right)$ and minimum $\left(\mathrm{E}_{v_{\mu}}^{2} \mathrm{~F}_{v_{\mu}}-2 \sigma\right)$ fluences for each GRB in the sample.

${ }^{5}$ The introduction of such a distribution in the analysis does not introduce any bias, as it can be shown that the Swift $z$-distribution is representative of the entire sample of long GRBs detected by any instrument from 1997 until today. 


\section{Signal and background estimation}

For each source in the sample, a Monte Carlo (MC) simulation of the expected neutrino signal is performed, while the respective background is estimated directly from off-source data collected by ANTARES. Only track-like events reconstructed within $10^{\circ}$ in radius from the expected GRB position and in temporal correlation with the duration of the prompt gamma-ray emission are selected, namely those falling within a search time window around the GRB occurrence $\left(T_{\text {search }}\right.$ $\mathrm{T}_{90}$ ). Both in signal and background estimations, the different environmental conditions in the deep sea and the variation of data-taking efficiency are taken into account, as explained in more details in [10].

\section{Analysis method}

The statistical analysis is built in order to maximise the chance of signal detection based on the internal shock model: a comparison between the expected signal and background probability density functions is needed in order to discriminate between both. This is achieved by considering respectively MC reconstructed track events from both $v_{\mu}-\bar{v}_{\mu}$ charged current interactions and hadronic showers for signal, while data are adopted for the latter. A strategy based on pseudoexperiments, simulating with high statistics a measurement's result, and on the optimisation of the cut on the track reconstruction quality maximising the Model Discovery Potential (MDP), is implemented. See [10] for further details.

\section{Results and conclusion of the stacking analysis}

ANTARES data from the end of 2007 to 2017 are analysed accordingly to the results of the optimisation procedure, searching for neutrino events in spatial and temporal coincidence with the prompt phase of GRBs. The optimal number of GRBs to stack was obtained, yielding the highest MDP at $3 \sigma$. Nonetheless, as a negligible reduction of the $\operatorname{MDP}_{3 \sigma}$ would have been obtained when stacking the entire catalogue, the flux from the whole sample of 784 GRBs was investigated, corresponding to a $\mathrm{MDP}_{3 \sigma}=0.03_{-0.02}^{+0.11}$ and a number of signal events $\mathrm{n}_{\mathrm{s}}\left(\mathrm{N}_{\mathrm{GRB}}=784\right)=0.03_{-0.02}^{+0.14}$. After unblinding ANTARES data, no event was found in spatial and temporal coincidence with the GRB sample to pass the selection criteria, for an equivalent livetime of the search of 18.9 hours. The absence of signal allows to derive $90 \%$ confidence level (CL) upper limits on the computed neutrino fluence, which read as $1.3_{-0.8}^{+4.1} \times 10^{-2} \mathrm{GeV} \mathrm{cm}^{-2}$ and $0.8_{-0.7}^{+5.2} \times 10^{-1} \mathrm{GeV} \mathrm{cm}^{-2}$, corresponding to $1.3_{-0.8}^{+0.4} \times 10^{-9} \mathrm{GeV} \mathrm{cm}^{-2} \mathrm{~s}^{-1} \mathrm{sr}^{-1}$ and $1.0_{-0.5}^{+0.9} \times 10^{-8} \mathrm{GeV} \mathrm{cm}^{-2} \mathrm{~s}^{-1} \mathrm{sr}^{-1}$, respectively, in terms of quasi-diffuse flux $\mathrm{E}_{v_{\mu}}^{2} \phi_{v_{\mu}}$ in the energy range from $\sim 60 \mathrm{TeV}$ to $\sim 10 \mathrm{PeV}$. The quasi-diffuse expected flux and corresponding upper limit, as calculated from the mean expected fluence, are shown in Fig. 4(a) and compared to previous ANTARES limits [5]. The results are also compared with the latest IceCube all-sky search [9], where no statistically significant signal from GRBs was found. Finally, the expected quasi-diffuse neutrino flux from the selected 784 GRBs and the corresponding upper limit are compared, in Fig. 4(b), with the diffuse astrophysical flux observed by IceCube $[21,22]$. From such a comparison, it is possible to conclude that, within standard assumptions of energy partition among accelerated hadrons, leptons and magnetic fields (i.e. baryonic loading 


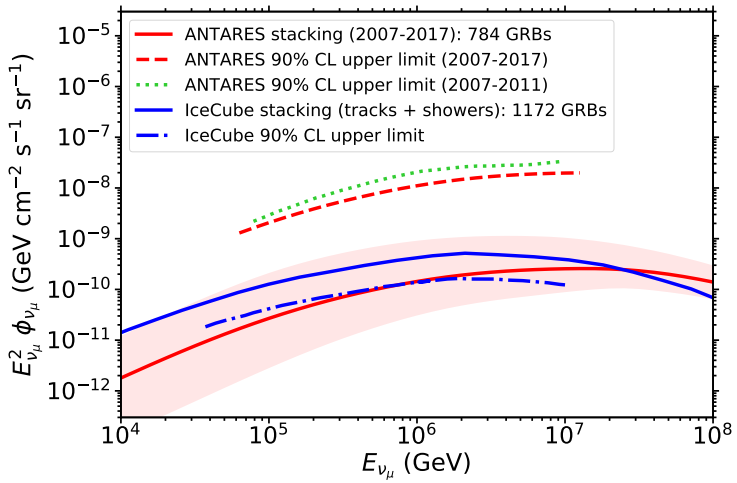

(a)

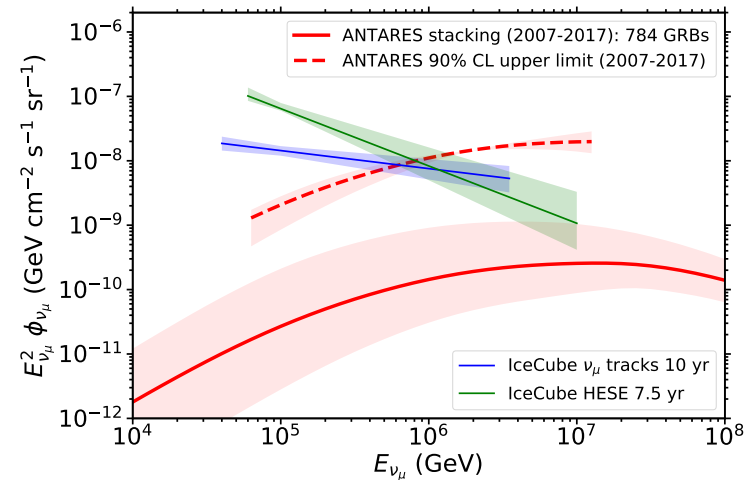

(b)

Figure 4: (a) Comparison between the ANTARES 90\% CL upper limit (red dashed line) with respect to the expected quasi-diffuse flux for 784 GRBs (red solid line) and the previous ANTARES 90\% CL upper limit (green dashed line) [5]. The solid blue line represents the quasi-diffuse flux expected from 1172 GRBs analysed by IceCube, while the dash-dotted blue line shows the corresponding IceCube 90\% CL upper limit [9]. (b) GRB quasi-diffuse flux expected for the 784 selected GRBs (red solid line) and the corresponding ANTARES 90\% CL upper limit (dashed red line). The red shaded regions show the uncertainty around the GRB quasi-diffuse flux, as in Fig. 3, and also around the computed upper limit. IceCube best fits for $v_{\mu}$ tracks in 10 years [21] and for HESE events in 7.5 years of collected data [22] are shown in blue and green, respectively.

equal to 10), GRBs are not the main sources of the astrophysical neutrino flux, possibly contributing less than $10 \%$ at energies around $100 \mathrm{TeV}$. This result confirms previous searches performed by IceCube [7-9]. Consequently, the parameter space still allowed to the internal shock model is characterised by sizeably smaller baryonic loading of GRB jets.

\section{References}

[1] Aartsen M. G. et al. (IceCube Collaboration), Evidence for High-Energy Extraterrestrial Neutrinos at the IceCube Detector Science 342 (2013) 1242856.

[2] Zhang B., Kumar P., The physics of gamma-ray bursts \& relativistic jets Phys. Rep. 561 (2015) 1.

[3] Ageron M. et al. (ANTARES Collaboration), ANTARES: the first undersea neutrino telescope Nucl. Instr. Meth. A 656 (2011) 11.

[4] Ahrens J. et al. (IceCube Collaboration), Sensitivity of the IceCube Detector to Astrophysical Sources of High Energy Muon Neutrinos Astropart. Phys. 20 (2004) 507.

[5] Adrián-Martínez S. et al. (ANTARES Collaboration), Search for muon neutrinos from gammaray bursts with the ANTARES neutrino telescope using 2008 to 2011 data A\&A 559 (2013) A9. 
[6] Adrián-Martínez S. et al. (ANTARES Collaboration), Search for high-energy neutrinos from bright GRBs with ANTARES MNRAS 469 (2017) 906.

[7] Aartsen M. G. et al. (IceCube Collaboration), Search for prompt neutrino emission from gamma-ray bursts with IceCube ApJ 805 (2015) L5.

[8] Aartsen M. G. et al. (IceCube Collaboration), An all-sky search for three flavors of neutrinos from gamma-ray bursts with the IceCube observatory ApJ 824 (2016) 115.

[9] Aartsen M. G. et al. (IceCube Collaboration), Extending the Search for Muon Neutrinos Coincident with Gamma-Ray Bursts in IceCube Data ApJ 843 (2017) 112.

[10] Albert A. et al. (ANTARES Collaboration), Constraining the contribution of Gamma-Ray Bursts to the high-energy diffuse neutrino flux with 10 yr of ANTARES data MNRAS $\mathbf{5 0 0}$ (2021) 5614.

[11] Ajello M. et al., (Fermi-LAT Collaboration), A Decade of Gamma-Ray Bursts Observed by Fermi-LAT: The Second GRB Catalog ApJ 878 (2019) 52.

[12] Hümmer S. et al., Simplified Models for Photohadronic Interactions in Cosmic Accelerators ApJ 721 (2010) 630.

[13] Hümmer S., Philipp B., Walter W., Neutrino Emission from Gamma-Ray Burst Fireballs, Revised Phys. Rev. Lett. 108 (2012) 231101.

[14] Rees M., Meśzaŕos P., Relativistic fireballs: energy conversion and time-scales MNRAS 258 (1992) 41.

[15] Lü J., Zou Y. C., Lei W. H., Lorentz-factor-Isotropic-luminosity/Energy Correlations of Gamma-Ray Bursts and Their Interpretation ApJ 751 (2012) 49.

[16] Golkhou V. Z., Butler N. R., Uncovering the intrinsic variability of Gamma-Ray Bursts ApJ 787 (2014) 90.

[17] Golkhou V. Z., Butler N. R., Littlejohns O. M., The Energy Dependence of GRB Minimum Variability Timescales ApJ 811 (2015) 93.

[18] Sonbas E. et al., Gamma-Ray Bursts: temporal scales and the bulk Lorentz factor ApJ 805 (2015) 86.

[19] He H.-N. et al., IceCube nondetection of Gamma-Ray Bursts: constraints on the fireball properties ApJ 752 (2012) 29.

[20] Ghirlanda G. et al., Gamma-ray bursts in the comoving frame MNRAS 420 (2012) 483.

[21] Stettner J. et al., Measurement of the Diffuse Astrophysical Muon-Neutrino Spectrum with Ten Years of IceCube Data PoS ICRC2019 (2019) 1017.

[22] Schneider A. et al., Characterization of the Astrophysical Diffuse Neutrino Flux with IceCube High-Energy Starting Events PoS ICRC2019 (2019) 1004. 


\section{Full Authors List: ANTARES Collaboration}

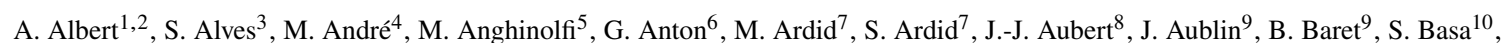
B. Belhorma ${ }^{11}$, M. Bendahman ${ }^{9,12}$, V. Bertin ${ }^{8}$, S. Biagi ${ }^{13}$, M. Bissinger $^{6}$, J. Boumaaza ${ }^{12}$, M. Bouta ${ }^{14}$, M.C. Bouwhuis ${ }^{15}$, H. Brânzaş ${ }^{16}$, R. Bruijn ${ }^{15,17}$, J. Brunner ${ }^{8}$, J. Busto ${ }^{8}$, B. Caiff ${ }^{5}$, A. Capone ${ }^{18,19}$, L. Caramete ${ }^{16}$, J. Carr ${ }^{8}$, V. Carretero ${ }^{3}$, S. Celli ${ }^{18,19}$, M. Chabab ${ }^{20}$, T. N. Chau ${ }^{9}$, R. Cherkaoui El Moursli ${ }^{12}$, T. Chiarusi ${ }^{21}$, M. Circella $^{22}$, A. Coleiro ${ }^{9}$, M. Colomer-Molla ${ }^{9,3}$, R. Coniglione ${ }^{13}$, P. Coyle $^{8}$, A. Creusot $^{9}$, A. F. Díaz ${ }^{23}$, G. de Wasseige ${ }^{9}$, A. Deschamps ${ }^{24}$, C. Distefano ${ }^{13}$, I. Di Palma ${ }^{18,19}$, A. Domi ${ }^{15,17}$, C. Donzaud ${ }^{9,25}$, D. Dornic ${ }^{8}$, D. Drouhin ${ }^{1,2}$, T. Eberl ${ }^{6}$, T. van Eeden ${ }^{15}$, D. van Eijk ${ }^{15}$, N. El Khayati ${ }^{12}$, A. Enzenhöfer ${ }^{8}$, P. Fermani ${ }^{18,19}$, G. Ferrara ${ }^{13}$, F. Filippini ${ }^{21,26}$, L.A. Fusco ${ }^{8}$, Y. Gatelet ${ }^{9}$, P. Gay ${ }^{27,9}$, H. Glotin ${ }^{28}$, R. Gozzini ${ }^{3}$, R. Gracia Ruiz ${ }^{15}$, K. Graf ${ }^{6}$, C. Guidi ${ }^{5,29}$, S. Hallmann 6 , H. van Haren ${ }^{30}$, A.J. Heijboer ${ }^{15}$, Y. Hello ${ }^{24}$, J.J. Hernández-Rey ${ }^{3}$, J. Hößl ${ }^{6}$, J. Hofestädt ${ }^{6}$, F. Huang ${ }^{8}$, G. Illuminati ${ }^{9}, 21,26$, C.W James ${ }^{31}$, B. Jisse-Jung ${ }^{15}$, M. de Jong ${ }^{15,32}$, P. de Jong ${ }^{15}$, M. Kadler ${ }^{33}$, O. Kalekin ${ }^{6}$, U. Katz ${ }^{6}$, N.R. Khan-Chowdhury ${ }^{3}$, A. Kouchner ${ }^{9}$, I. Kreykenbohm ${ }^{34}$, V. Kulikovskiy ${ }^{5,36}$, R. Lahmann ${ }^{6}$, R. Le Breton ${ }^{9}$, D. Lefèvre ${ }^{35}$, E. Leonora ${ }^{36}$, G. Levi ${ }^{21,26}$, M. Lincetto ${ }^{8}$, D. Lopez-Coto ${ }^{37}$, S. Loucatos ${ }^{38,9}$, L. Maderer ${ }^{9}$, J. Manczak ${ }^{3}$, M. Marcelin ${ }^{10}$, A. Margiotta ${ }^{21,26}$, A. Marinelli ${ }^{39}$, J.A. Martínez-Mora ${ }^{7}$, K. Melis ${ }^{15,17}$, P. Migliozzi ${ }^{39}$, A. Moussa ${ }^{14}$, R. Muller ${ }^{15}$, L. Nauta ${ }^{15}$, S. Navas ${ }^{37}$, E. Nezri ${ }^{10}$, B. O' Fearraigh $^{15}$, A. Păun ${ }^{16}$, G.E. Păvălaş ${ }^{16}$, C. Pellegrino ${ }^{21,40,41}$, M. Perrin-Terrin ${ }^{8}$, V. Pestel ${ }^{15}$, P. Piattelli ${ }^{13}$, C. Pieterse ${ }^{3}$, C. Poirè ${ }^{7}$, V. Popa ${ }^{16}$, T. Pradier ${ }^{1}$, N. Randazzo ${ }^{36}$, S. Reck ${ }^{6}$, G. Riccobene ${ }^{13}$, A. Romanov ${ }^{5,29}$, A. Sánchez-Losa ${ }^{3,22}$, F. Salesa Greus ${ }^{3}$, D. F. E. Samtleben ${ }^{15,32}$, M. Sanguineti ${ }^{5,29}$, P. Sapienza ${ }^{13}$, J. Schnabel ${ }^{6}$, J. Schumann ${ }^{6}$, F. Schüssler ${ }^{38}$, M. Spurio ${ }^{21,26}$, Th. Stolarczyk ${ }^{38}$, M. Taiuti ${ }^{5,29}$, Y. Tayalati ${ }^{12}$, S.J. Tingay ${ }^{31}$, B. Vallage ${ }^{38,9}$, V. Van Elewyck ${ }^{9,41}$, F. Versari ${ }^{21,26,9}$, S. Viola ${ }^{13}$, D. Vivolo ${ }^{39,43}$, J. Wilms ${ }^{34}$, S. Zavatarelli ${ }^{5}$, A. Zegarelli ${ }^{18,19}$, J.D. Zornoza ${ }^{3}$, and J. Zúñiga ${ }^{3}$

${ }^{1}$ Université de Strasbourg, CNRS, IPHC UMR 7178, F-67000 Strasbourg, France. ${ }^{2}$ Université de Haute Alsace, F-68100 Mulhouse, France. ${ }^{3}$ IFIC - Instituto de Física Corpuscular (CSIC - Universitat de València) c/ Catedrático José Beltrán, 2 E-46980 Paterna, Valencia, Spain. ${ }^{4}$ Technical University of Catalonia, Laboratory of Applied Bioacoustics, Rambla Exposició, 08800 Vilanova i la Geltrú, Barcelona, Spain. ${ }^{5}$ INFN - Sezione di Genova, Via Dodecaneso 33, 16146 Genova, Italy. ${ }^{6}$ Friedrich-Alexander-Universität Erlangen-Nürnberg, Erlangen Centre for Astroparticle Physics, Erwin-Rommel-Str. 1, 91058 Erlangen, Germany. ${ }^{7}$ Institut d'Investigació per a la Gestió Integrada de les Zones Costaneres (IGIC) - Universitat Politècnica de València. C/ Paranimf 1, 46730 Gandia, Spain. ${ }^{8}$ Aix Marseille Univ, CNRS/IN2P3, CPPM, Marseille, France. ${ }^{9}$ Université de Paris, CNRS, Astroparticule et Cosmologie, F-75013 Paris, France. ${ }^{10}$ Aix Marseille Univ, CNRS, CNES, LAM, Marseille, France. ${ }^{11}$ National Center for Energy Sciences and Nuclear Techniques, B.P.1382, R. P.10001 Rabat, Morocco. ${ }^{12}$ University Mohammed V in Rabat, Faculty of Sciences, 4 av. Ibn Battouta, B.P. 1014, R.P. 10000 Rabat, Morocco. ${ }^{13}$ INFN - Laboratori Nazionali del Sud (LNS), Via S. Sofia 62, 95123 Catania, Italy. ${ }^{14}$ University Mohammed I, Laboratory of Physics of Matter and Radiations, B.P.717, Oujda 6000, Morocco. ${ }^{15}$ Nikhef, Science Park, Amsterdam, The Netherlands. ${ }^{16}$ Institute of Space Science, RO-077125 Bucharest, Măgurele, Romania. ${ }^{17}$ Universiteit van Amsterdam, Instituut voor Hoge-Energie Fysica, Science Park 105, 1098 XG Amsterdam, The Netherlands. ${ }^{18}$ INFN - Sezione di Roma, P.le Aldo Moro 2, 00185 Roma, Italy. ${ }^{19}$ Dipartimento di Fisica dell'Università La Sapienza, P.le Aldo Moro 2, 00185 Roma, Italy. ${ }^{20}$ LPHEA, Faculty of Science - Semlali, Cadi Ayyad University, P.O.B. 2390, Marrakech, Morocco. ${ }^{21}$ INFN - Sezione di Bologna, Viale Berti-Pichat 6/2, 40127 Bologna, Italy. ${ }^{22}$ INFN - Sezione di Bari, Via E. Orabona 4, 70126 Bari, Italy. ${ }^{23}$ Department of Computer Architecture and Technology/CITIC, University of Granada, 18071 Granada, Spain. ${ }^{24}$ Géoazur, UCA, CNRS, IRD, Observatoire de la Côte d'Azur, Sophia Antipolis, France. ${ }^{25}$ Université Paris-Sud, 91405 Orsay Cedex, France. ${ }^{26}$ Dipartimento di Fisica e Astronomia dell'Università, Viale Berti Pichat 6/2, 40127 Bologna, Italy. ${ }^{27}$ Laboratoire de Physique Corpusculaire, Clermont Université, Université Blaise Pascal, CNRS/IN2P3, BP 10448, F-63000 Clermont-Ferrand, France. ${ }^{28}$ LIS, UMR Université de Toulon, Aix Marseille Université, CNRS, 83041 Toulon, France. ${ }^{29}$ Dipartimento di Fisica dell'Università, Via Dodecaneso 33, 16146 Genova, Italy. ${ }^{30}$ Royal Netherlands Institute for Sea Research (NIOZ), Landsdiep 4, 1797 SZ 't Horntje (Texel), the Netherlands. ${ }^{31}$ International Centre for Radio Astronomy Research - Curtin University, Bentley, WA 6102, Australia. ${ }^{32}$ Huygens-Kamerlingh Onnes Laboratorium, Universiteit Leiden, The Netherlands. ${ }^{33}$ Institut für Theoretische Physik und Astrophysik, Universität Würzburg, Emil-Fischer Str. 31, 97074 Würzburg, Germany. ${ }^{34}$ Dr. Remeis-Sternwarte and ECAP, Friedrich-Alexander-Universität Erlangen-Nürnberg, Sternwartstr. 7, 96049 Bamberg, Germany. ${ }^{35}$ Mediterranean Institute of Oceanography (MIO), Aix-Marseille University, 13288, Marseille, Cedex 9, France; Université du Sud Toulon-Var, CNRS-INSU/IRD UM 110, 83957, La Garde Cedex, France. ${ }^{36}$ INFN - Sezione di Catania, Via S. Sofia 64, 95123 Catania, Italy. ${ }^{37}$ Dpto. de Física Teórica y del Cosmos \& C.A.F.P.E., University of Granada, 18071 Granada, Spain. ${ }^{38}$ IRFU, CEA, Université Paris-Saclay, F-91191 Gif-sur-Yvette, France. ${ }^{39}$ INFN - Sezione di Napoli, Via Cintia 80126 Napoli, Italy. ${ }^{40}$ Museo Storico della Fisica e Centro Studi e Ricerche Enrico Fermi, Piazza del Viminale 1, 00184, Roma. ${ }^{41}$ INFN - CNAF, Viale C. Berti Pichat 6/2, 40127, Bologna. ${ }^{42}$ Institut Universitaire de France, 75005 Paris, France. ${ }^{43}$ Dipartimento di Fisica dell’Università Federico II di Napoli, Via Cintia 80126, Napoli, Italy. 